\title{
New Approximations to the Mathematical Constant $e$
}

\author{
Sanjay Kumar Khattri (Corresponding author) \\ Stord Haugesund University College \\ Bjørnsonsgate 45 \\ PO box 5528, Haugesund, Norway \\ Tel: 47-5270-2683 E-mail: sanjay.khattri@hsh.no
}

\begin{abstract}
Based on the Newton-Cotes and Gaussian quadrature rules, we develop several new closed form approximations to the mathematical constant $e$. For validating effectiveness of our approximations, a comparison of our results to the existing approximations is also presented. Because of the level of mathematics, the presented work is easily embraceable in an undergraduate class. Another aim of this work is to encourage students for formulating other better approximations by using the suggested strategy.
\end{abstract}

Keywords: Mathematical constant, Closed form approximation, Quadrature

\section{Introduction}

The number $e$ is one of the most fundamental numbers in mathematics. This number is also referred to as Euler's number or Napier's constant. In this work, we develop several new closed form approximations to the mathematical constant $e$ through quadrature rules. Classically the number $e$ is defined as

$$
e \stackrel{\text { def }}{=} \lim _{n \rightarrow \infty}\left(1+\frac{1}{n}\right)^{n}
$$

Let us call this definition the Euler's e (Ee).

First year undergraduate students are exposed to concepts of limits and quadrature. By using these concepts, we are further refining the limit (1). Based on this work, teacher can ask students to formulate even better approximations to the mathematical constant $e$.

Figure 1 presents a graph of the function $1 / x$. The area under the graph and between the vertical lines $x=n$ and $x=n+1$ is given as

$$
\int_{n}^{n+1} \frac{1}{x} \mathrm{~d} x
$$

For forming various closed form approximations to $e$, we use quadrature rule for approximating the integral. The exact value of this integral is $\ln \left(1+\frac{1}{n}\right)$.

\section{Approximation through trapezoidal quadrature rule}

The Trapezoidal quadrature rule is given as

$$
\int_{n}^{n+1} \frac{1}{x} \mathrm{~d} x=\frac{h}{2}\left[f\left(x_{1}\right)+f\left(x_{2}\right)\right]
$$


Here, $h=1, x_{1}=n$ and $x_{2}=n+1$. Thus,

$$
\begin{aligned}
{[\ln x]_{n}^{n+1} } & =\frac{1}{2}\left[\frac{1}{n}+\frac{1}{n+1}\right] \\
\ln \left(\frac{n+1}{n}\right) & =\frac{1}{n}\left[\frac{n+\frac{1}{2}}{n+1}\right] \\
& =\frac{1}{n}\left[\frac{1}{1+\frac{0.5}{n+0.5}}\right] \\
\Rightarrow n\left[1+\frac{0.5}{n+0.5}\right] \ln \left(\frac{n+1}{n}\right) & =1 \\
\ln \left(\frac{n+1}{n}\right)^{n}\left[1+\frac{0.5}{n+0.5}\right] & =\ln e \\
\Rightarrow e=\left(\frac{n+1}{n}\right)^{n}\left[1+\frac{0.5}{n+0.5}\right] &
\end{aligned}
$$

The definition of $e$ through the trapezoidal rule is given as

$$
e \stackrel{\text { def }}{=} \lim _{n \rightarrow \infty}\left(\frac{n+1}{n}\right)^{n}\left[1+\frac{0.5}{n+0.5}\right]
$$

Let us call this definition, the Trapezoidal Euler's e (TEE).

\section{Approximation through Simpson's quadrature rule}

The Simpson's $\frac{1}{3}$ quadrature rule for approximating integral is given as

$$
\int_{n}^{n+1} \frac{1}{x} \mathrm{~d} x=\frac{h}{3}\left[f\left(x_{0}\right)+4 f\left(x_{1}\right)+f\left(x_{2}\right)\right]
$$

Here, $h=\frac{1}{2}, x_{0}=n, x_{1}=\frac{2 n+1}{2}$ and $x_{2}=n+1$

$$
\begin{aligned}
\int_{n}^{n+1} \frac{1}{x} \mathrm{~d} x & =\frac{1}{6}\left[\frac{1}{n}+\frac{8}{2 n+1}+\frac{1}{n+1}\right] \\
\ln \left(\frac{n+1}{n}\right) & =\frac{1}{n\left(1+\frac{6 n+5}{12 n^{2}+12 n+1}\right)} \\
\Rightarrow n\left(1+\frac{6 n+5}{12 n^{2}+12 n+1}\right) \ln \left(1+\frac{1}{n}\right) & =1 \\
\ln \left(1+\frac{1}{n}\right)^{n\left[1+\frac{6 n+5}{12 n^{2}+12 n+1}\right]} & =\ln e \\
\Rightarrow e=\left(1+\frac{1}{n}\right)^{n\left[1+\left(\frac{6 n+5}{12 n^{2}+12 n+1}\right)\right]} &
\end{aligned}
$$

The definition of $e$ through the Simpson's quadrature rule is

$$
e \stackrel{\text { def }}{=} \lim _{n \rightarrow \infty}\left(1+\frac{1}{n}\right)^{n\left[1+\left(\frac{6 n+5}{12 n^{2}+12 n+1}\right)\right]}
$$

Let us call this definition, the $\frac{1}{3}$ Simpson Euler's $e$ ( $\left.\frac{1}{3} \mathrm{SEE}\right)$ 


\section{Approximation through Simpson's $\frac{3}{8}$ quadrature rule}

Approximation of the integral through Simpson's $\frac{3}{8}$ quadrature rule is

$$
\int_{n}^{n+1} \frac{1}{x} \mathrm{~d} x=\frac{3 h}{8}\left[f\left(x_{0}\right)+3 f\left(x_{1}\right)+3 f\left(x_{2}\right)+f\left(x_{3}\right)\right]
$$

Here, $h=\frac{1}{3}, x_{0}=n, x_{1}=\frac{3 n+1}{3}, x_{2}=\frac{3 n+2}{3}$ and $x_{3}=n+1$

$$
\begin{aligned}
& \int_{n}^{n+1} \frac{1}{x} \mathrm{~d} x=\frac{1}{n}\left[\frac{36 n^{3}+54 n^{2}+20 n+1}{\left(36 n^{3}+54 n^{2}+20 n+1\right)+18 n^{2}+24 n+7}\right] \\
& \ln \left(1+\frac{1}{n}\right)=\frac{1}{n}\left[\frac{1}{1+\left(\frac{18 n^{2}+24 n+7}{36 n^{3}+54 n^{2}+20 n+1}\right)}\right] \\
& \Rightarrow n\left[1+\left(\frac{18 n^{2}+24 n+7}{36 n^{3}+54 n^{2}+20 n+1}\right)\right] \ln \left(1+\frac{1}{n}\right)=1 \\
& \ln \left(1+\frac{1}{n}\right)^{n\left[1+\left(\frac{18 n^{2}+24 n+7}{36 n^{3}+54 n^{2}+20 n+1}\right)\right]}=\ln e \\
& \Rightarrow e=\left(1+\frac{1}{n}\right)^{n\left[1+\left(\frac{18 n^{2}+24 n+7}{36 n^{3}+54 n^{2}+20 n+1}\right)\right]}
\end{aligned}
$$

The definition of $e$ through the Simpson's $\frac{3}{8}$ quadrature rule is

$$
e \stackrel{\text { def }}{=} \lim _{n \rightarrow \infty}\left(1+\frac{1}{n}\right)^{n\left[1+\left(\frac{18 n^{2}+24 n+7}{36 n^{3}+54 n^{2}+20 n+1}\right)\right]}
$$

Let us call this definition, the $\frac{3}{8}$ Simpson Euler's $e\left(\frac{3}{8} \mathrm{SEE}\right)$.

\section{Approximating $e$ through Boole's quadrature rule}

The Boole's quadrature rule is given as follows

$$
\int_{n}^{n+1} \frac{1}{x} \mathrm{~d} x=\frac{2 h}{45}\left[7 f\left(x_{0}\right)+32 f\left(x_{1}\right)+12 f\left(x_{2}\right)+32 f\left(x_{3}\right)+7 f\left(x_{4}\right)\right]
$$

Here, $h=\frac{1}{4}, x_{0}=n, x_{1}=\frac{4 n+1}{4}, x_{2}=\frac{4 n+2}{4}, x_{3}=\frac{4 n+3}{4}$ and $x_{4}=n+1$

$$
\begin{gathered}
\ln \left(1+\frac{1}{n}\right)=\frac{2}{4 \times 45}\left[\frac{7}{n}+\frac{32 \times 4}{4 n+1}+\frac{12 \times 4}{4 n+2}+\frac{32 \times 4}{4 n+3}+\frac{7}{n+1}\right] \\
=\frac{1}{n}\left[\frac{1}{\left.1+\left(\frac{480 n^{3}+880 n^{2}+490 n+83}{960 n^{4}+1920 n^{3}+1220 n^{2}+260 n+7}\right)\right]}\right. \\
n\left[1+\left(\frac{480 n^{3}+880 n^{2}+490 n+83}{960 n^{4}+1920 n^{3}+1220 n^{2}+260 n+7}\right)\right] \ln \left(1+\frac{1}{n}\right)=1 \\
\Rightarrow e=\left(1+\frac{1}{n}\right)^{n}\left[1+\left(\frac{480 n^{3}+880 n^{2}+400 n+83}{960 n^{2}+1920 n^{3}+1220 n^{2}+260 n+7}\right)\right]
\end{gathered}
$$


The definition of $e$ through the Boole's quadrature rule is

$$
e \stackrel{\text { def }}{=} \lim _{n \rightarrow \infty}\left(1+\frac{1}{n}\right)^{n\left[1+\left(\frac{480 n^{3}+880 n^{2}+490 n+83}{960 n^{4}+1920 n^{3}+1220 n^{2}+260 n+7}\right)\right]}
$$

Let us call this definition, the Boole Euler's $e$ (BEE).

\section{Approximating $e$ through Gauss-Legendre 2 point quadrature}

The two point Gauss-Legendre Quadrature is given as

$$
\int_{n}^{n+1} \frac{1}{x} \mathrm{~d} x=k\left[w_{1} f\left(x_{1}\right)+w_{2} f\left(x_{2}\right)\right]
$$

Here, $k=\frac{n+1-n}{2}=\frac{1}{2}, x_{1}=\frac{2 n+1}{2}+\frac{1}{2 \sqrt{3}}$ and $x_{3}=\frac{2 n+1}{2}-\frac{1}{2 \sqrt{3}}$. Weights are $w_{1}=1$ and $w_{2}=1$.

$$
\begin{aligned}
\int_{n}^{n+1} \frac{1}{x} \mathrm{~d} x & =\frac{1}{2}\left[\frac{2 \sqrt{3}}{(2 n+1) \sqrt{3}+1}+\frac{2 \sqrt{3}}{(2 n+1) \sqrt{3}-1}\right] \\
\ln \left(1+\frac{1}{n}\right) & =\frac{6 n+3}{6 n^{2}+6 n+1} \\
\frac{6 n^{2}+6 n+1}{6 n+3} \ln \left(1+\frac{1}{n}\right) & =1 \\
\ln \left(1+\frac{1}{n}\right)^{\frac{6 n^{2}+6 n+1}{6 n+3}} & =\ln e \\
\Rightarrow e & =\left(1+\frac{1}{n}\right)^{\frac{6 n^{2}+6 n+1}{6 n+3}} \\
e & =\left(1+\frac{1}{n}\right)^{n\left[1+\left(\frac{3+\frac{1}{n}}{6 n+3}\right)\right]}
\end{aligned}
$$

The definition of $e$ through the two point Gauss-Legendre quadrature rule is

$$
e \stackrel{\text { def }}{=} \lim _{n \rightarrow \infty}\left(1+\frac{1}{n}\right)^{n}\left[1+\left(\frac{3+\frac{1}{n}}{6 n+3}\right)\right]
$$

Let us call this definition, the two point Gauss-Legendre Euler's $e$ (2P-GLEE).

\section{Approximating $e$ through Gauss-Legendre 3 point quadrature}

Three point Gauss-Legendre quadrature rule is given as

$$
\int_{n}^{n+1} \frac{1}{x} \mathrm{~d} x=k\left[w_{1} f\left(x_{1}\right)+w_{2} f\left(x_{2}\right)+w_{3} f\left(x_{3}\right)\right]
$$

The weights $w_{i}$ and points $x_{i}$ are given as

$$
\begin{array}{ll}
w_{1}=\frac{8}{9} & x_{1}=\frac{2 n+1}{2} \\
w_{2}=\frac{5}{9} & x_{2}=\frac{(2 n+1) \sqrt{5}+\sqrt{3}}{2 \sqrt{5}} \\
w_{3}=\frac{5}{9} & x_{3}=\frac{(2 n+1) \sqrt{5}-\sqrt{3}}{2 \sqrt{5}}
\end{array}
$$


Thus,

$$
\begin{aligned}
& \ln \left(1+\frac{1}{n}\right)=\frac{1}{9}\left[6 \frac{60 n^{2}+60 n+11}{(2 n+1)(2 \sqrt{5} n+\sqrt{5}+\sqrt{3})(2 \sqrt{5} n+\sqrt{5}-\sqrt{3})}\right] \\
& \ln \left(1+\frac{1}{n}\right)=\left[\frac{\left(60 n^{2}+60 n+11\right)}{60 n^{3}+90 n^{2}+36 n+3}\right] \\
& =\frac{1}{n}\left[\frac{1}{1+\left(\frac{30 n+25+\frac{3}{n}}{60 n^{2}+60 n+11}\right)}\right] \\
& n\left[1+\left(\frac{30 n+25+\frac{3}{n}}{60 n^{2}+60 n+11}\right)\right] \ln \left(1+\frac{1}{n}\right)=1 \\
& \ln \left(1+\frac{1}{n}\right)^{n\left[1+\left(\frac{30 n+25+\frac{3}{n}}{60 n^{2}+60 n+11}\right)\right]}=\ln e \\
& \Rightarrow \quad e=\left(1+\frac{1}{n}\right)^{n\left[1+\frac{30 n+25+\frac{3}{n}}{60 n^{2}+60 n+11}\right]}
\end{aligned}
$$

The definition of $e$ through the three point Gauss-Legendre quadrature rule is

$$
e \stackrel{\text { def }}{=} \lim _{n \rightarrow \infty}\left(1+\frac{1}{n}\right)^{n\left[1+\frac{30 n+25+\frac{3}{n}}{60 n^{2}+60 n+11}\right]}
$$

Let us call this definition, the three point Gauss-Legendre Euler's $e$ (3P-GLEE). The English meaning of the word "GLEE"is brightness. Through numerical work, we will see that it is indeed a very bright approximation to the fundamental constant $e$. For $n=100$, the 3P-GLEE gives us "2.71828182845904617759", and which is $e$ accurate to fifteen decimal places. If we replace $n$ by $2^{n}$ as done by Knox (1999) and Brothers (1998) in their approximation formulae, then 3P-GLEE gives exact $e$ for $n=1109$.

\section{Approximating $e$ through Gauss-Legendre 4 point quadrature}

The four point Gauss-Legendre quadrature rule is given as

$$
\int_{n}^{n+1} \frac{1}{x} \mathrm{~d} x=k\left[w_{1} f\left(x_{1}\right)+w_{2} f\left(x_{2}\right)+w_{3} f\left(x_{3}\right)+w_{4} f\left(x_{4}\right)\right]
$$

Here, $k=\frac{1}{2}$. Weights $w_{i}$ and points $x_{i}$ are given as

$$
\begin{array}{ll}
w_{1}=\frac{18+\sqrt{30}}{36} & x_{1}=\frac{(2 n+1) \sqrt{7}+\sqrt{\left(3-2 \sqrt{\frac{6}{5}}\right)}}{2 \sqrt{7}} \\
w_{2}=\frac{18+\sqrt{30}}{36} & x_{2}=\frac{(2 n+1) \sqrt{7}-\sqrt{\left(3-2 \sqrt{\frac{6}{5}}\right)}}{2 \sqrt{7}} \\
w_{3}=\frac{18-\sqrt{30}}{36} & x_{3}=\frac{(2 n+1) \sqrt{7}+\sqrt{\left(3+2 \sqrt{\frac{6}{5}}\right)}}{2 \sqrt{7}} \\
w_{4}=\frac{18-\sqrt{30}}{36} & x_{4}=\frac{(2 n+1) \sqrt{7}-\sqrt{\left(3-2 \sqrt{\frac{6}{5}}\right)}}{2 \sqrt{7}}
\end{array}
$$


Thus,

$$
\begin{aligned}
\ln \left(1+\frac{1}{n}\right) & =\frac{420 n^{3}+630 n^{2}+260 n+25}{420 n^{4}+840 n^{3}+540 n^{2}+120 n+6} \\
& =\frac{1}{n}\left[\frac{420 n^{3}+630 n^{2}+260 n+25}{420 n^{3}+840 n^{2}+540 n+120+\frac{6}{n}}\right] \\
& =\frac{1}{n}\left[\frac{1}{1+\frac{210 n^{2}+280 n+95+6 n^{-1}}{420 n^{3}+630 n^{2}+260 n+25}}\right]
\end{aligned}
$$

Thus,

$$
\begin{aligned}
n\left[1+\frac{210 n^{2}+280 n+95+6 n^{-1}}{420 n^{3}+630 n^{2}+260 n+25}\right] \ln \left(1+\frac{1}{n}\right) & =1 \\
\ln \left(1+\frac{1}{n}\right)^{n}\left[1+\frac{210 n^{2}+280 n+95+6 n^{-1}}{420 n^{3}+630 n^{2}+260 n+25}\right] & =\ln e
\end{aligned}
$$

The definition of $e$ through the four point Gauss-Legendre quadrature rule is

$$
e \stackrel{\text { def }}{=} \lim _{n \rightarrow \infty}\left(1+\frac{1}{n}\right)^{n\left[1+\frac{210 n^{2}+280 n+95+6 n^{-1}}{420 n^{3}+630 n^{2}+260 n+25}\right]}
$$

Let us call this definition, the four point Gauss-Legendre Euler's $e$ (4P-GLEE). For $n=100$, the 4P-GLEE gives us "2.718281828459045235366210" and which is $e$ accurate to twenty one decimal places. If we replace $n$ by $2^{n}$ as done by Knox (1999) and Brothers (1998) in their approximation formulae, then 4P-GLEE gives exact $e$ for $n=832$.

\section{Approximating $e$ through Gauss-Legendre 5 point quadrature}

The five point Gauss-Legendre quadrature rule is given as:

$$
\int_{n}^{n+1} \frac{1}{x} \mathrm{~d} x=k\left[w_{1} f\left(x_{1}\right)+w_{2} f\left(x_{2}\right)+w_{3} f\left(x_{3}\right)+w_{4} f\left(x_{4}\right)+w_{5} f\left(x_{5}\right)\right]
$$

Here, $k=\frac{1}{2}$. Weights $w_{i}$ and points $x_{i}$ are given as

$$
\begin{aligned}
w_{1} & =\frac{128}{225} \quad x_{1}=n+\frac{1}{2} \\
w_{2} & =\frac{161}{450}+\frac{13}{900} \sqrt{70} \quad x_{2}=n+\frac{1}{2}+\frac{1}{42} \sqrt{245-14 \sqrt{70}} \\
w_{3} & =\frac{161}{450}+\frac{13}{900} \sqrt{70} \quad x_{3}=n+\frac{1}{2}-\frac{1}{42} \sqrt{245-14 \sqrt{70}} \\
w_{4} & =\frac{161}{450}+\frac{13}{900} \sqrt{70} \quad x_{4}=n+\frac{1}{2}+\frac{1}{42} \sqrt{245+14 \sqrt{70}} \\
w_{5} & =\frac{161}{450}-\frac{13}{900} \sqrt{70} \quad x_{5}=n+\frac{1}{2}-\frac{1}{42} \sqrt{245+14 \sqrt{70}} \\
\ln \left(1+\frac{1}{n}\right) & =\frac{7560 n^{4}+15120 n^{3}+9870 n^{2}+2310 n+137}{7560 n^{5}+18900 n^{4}+16800 n^{3}+6300 n^{2}+900 n+30} \\
& =\frac{1}{n}\left[\frac{7560 n^{4}+15120 n^{3}+9870 n^{2}+2310 n+137}{7560 n^{4}+18900 n^{3}+16800 n^{2}+6300 n+900+30 n^{-1}}\right] \\
& =\frac{1}{n}\left[\frac{1}{1+\frac{3780 n^{3}+6930 n^{2}+3990 n+763+30 n^{-1}}{7560 n^{4}+15120 n^{3}+9870 n^{2}+2310 n+137}}\right]
\end{aligned}
$$


Thus,

$$
\begin{array}{r}
n\left[1+\frac{3780 n^{3}+6930 n^{2}+3990 n+763+30 n^{-1}}{7560 n^{4}+15120 n^{3}+9870 n^{2}+2310 n+137}\right] \ln \left(1+\frac{1}{n}\right)=\ln e \\
\ln \left(1+\frac{1}{n}\right)^{n}\left[1+\frac{3780 n^{3}+6930 n^{2}+3990 n+763+30 n^{-1}}{7560 n^{4}+15120 n^{3}+9870 n^{2}+2310 n+137}\right]=\ln e
\end{array}
$$

The definition of $e$ through the five point Gauss-Legendre quadrature rule is

$$
e \stackrel{\text { def }}{=} \lim _{n \rightarrow \infty}\left(1+\frac{1}{n}\right)^{n\left[1+\frac{3780 n^{3}+6930 n^{2}+3990 n+763+30 n^{-1}}{7560 n^{4}+15120 n^{3}+9870 n^{2}+2310 n+137}\right]}
$$

Let us call this definition, the five point Gauss-Legendre Euler's $e$ (5P-GLEE). For $n=100$, the 5P-GLEE gives us "2.718281828459045235360287508375", and which is $e$ accurate to twenty five decimal places. On the other hand, the classical definition (1) gives $e$ accurate only to two decimal places.

Approximation by various “GLEE”formulae and Euler's e (Ee) equation (??) for $n=100$.

$$
e=\underbrace{\underbrace{\overbrace{\text { Ee }}^{2.7} 1828182845904523536}_{\text {3P-GLEE }} 0287}_{\text {SP-GLEE }} 47135266249775724709369996
$$

If we replace $n$ by $2^{n}$ as done by Knox (1999) and Brothers (1998), then 5P-GLEE gives exact $e$ for $n=666$.

After introducing students to the standard definition of the number $e$ given by equation (1). When we presented our new definitions and their derivations in the class, the students has shown considerable interest. Students find it very appealing that simple techniques gives us the closed form approximation which improves accuracy from two digits to twenty five digits.

\section{Numerical Work}

For performing computations to high accuracy, we are using the $\mathrm{C}^{++}$library ARPREC (D. H. Bailey, 2002). Let us now briefly mention existing relations for representing mathematical constant $e$. Knox (1999) and Brothers (1998) have also developed some very nice closed form approximations to the mathematical constant $e$. The Table (1) displays closed form formulae developed in Knox (1999) and Brothers (1998). Reader can observe that the formulae B1, B2, B3, B4, B5, B6 and $\mathbf{B} 7$ are not defined for $n=1$. On the other hand, formulae (2), (3), (4), (5), (6), (7), (8) and (9) are defined for $n=1$.

Let us now compare our formulae with the formulae presented in the Table 1. For $n=100$, Table 2 presents error in approximating $e$ through different closed form approximations. Here, error is equal to the exact value of the mathematical constant $e$ minus the value given by different approximations.

From the Table 2, it can be inferred that the approximations developed by us are more accurate. It is also obvious that our formulae are computationally efficient. For example, in the Table 1 the formula $B 7$ gives most accurate approximation. The reader can see that for evaluating $B 7$, we need to evaluate $B 6$ and $B 5$. And, for computing $B 6$, we need to compute $B 3$ and $B 4$. And for computing $B 4$, we need to compute $A C M$ and $B 2$. And so on.

In the different definitions of the constant $e$ through formulae (2), (3), (4), (5), (6), (7), (8) and (9). It can be seen that for large values of $n$, all of these formulae behaves as:

$$
e=\lim _{n \rightarrow \infty}\left(1+\frac{1}{n}\right)^{n+0.5}
$$

Let us call this definition, the Gauss $e(\mathbf{G E})$. The reader can observe that the $\mathbf{G E}$ is modestly different than the classical definition (1). To see why this definition of $e$ is more accurate than the classical definition of $e$. Let us compute $e$ from these two definitions for $n=1000$. From the classical definition, we get $e=2,71692393$. Which is accurate only till 3 decimal places. While from GE we get $e=2,71828205$. And, which is $e$ accurate till 6 decimal places. It is indeed a substantial improvement over classical result. We are just changing the classical definition slightly.

Let us now observe an interesting connection between the formula MIM (see Table 1) and our formulae GE (10). For $n=1000$, MIM gives $e=2,7182827346$. While from GE we get $e=2,71828205$. Both of these values are accurate till 6 decimal places. Thus, both of these formulae are giving same order of accuracy. This lead us to believe that they must be the same formulae. The readers are encouraged to see it for themselves. Replacing $n$ by $\left(\frac{n}{2}-0.5\right)$ in $\mathbf{G E}(10)$, we 
get MIM. These formulae are derived from different methods. The MIM is derived in Knox (1999) and Brothers (1998) by infinite series expansion. While, we obtained GE (10) from quadrature rules.

\section{Conclusions}

We have developed several closed form approximations to the mathematical constant $e$. Numerical comparison study validate the effectiveness of our results over the existing closed form approximations. The other main aim of this work is to encourage undergraduate students for developing new approximations. The strategy presented in this paper is easily adoptable in an undergraduate class. Based on the work presented in this paper, teachers can ask students to further develop new approximations by using various other quadratures. Through our teaching experience we found our work is encouraging students to formulate even better approximations to the constant $e$.

\section{References}

C. L. Wang. (1989). Simple Inequalities And Old Limits. American Mathematical Monthly. Vol. 96(4), April.

C. W. Barnes. (1984). Euler's constant and e. American Mathematical Monthly. Vol. 96(7).

D. H. Bailey, Y. Hida, X. S. Li and B. Thompson. (2002). ARPREC: An Arbitrary Precision Computation Package. LBNL-53651. Sept.

E. Maor. (1994). e: The Story of a Number. Princeton University Press..

H. J. Brothers and J. A. Knox. (1998). New closed-form approximations to the logarithmic constant e. Mathematical Intelligencer. 20(4).

H. Yang and H. Yang. (2001). The Arithmetic-Geometric Mean Inequality and the Constant e. Mathematics Magazine. Vol. 74(4) October.

J. A. Knox and H. J. Brothers. (1999). Novel series based approximations to e. The College Mathematics Journal, Vol. 30 (4), September.

R. Johnsonbaugh. (1981). The Trapezoid Rule, Stirling's Formula and Euler's constant. American Mathematical Monthly. Vol. 88(9), Nov. 1981.

T. N. T. Goodman. (1986). Maximum products and $\lim \left(1+\frac{1}{n}\right)^{n}=$ e. American Mathematical Monthly. Vol. 93(9), October 1986. 
Table 1. Different closed form approximations to the number $e$ from Knox (1999) and Brothers (1998).

\begin{tabular}{|c|c|}
\hline Formulae & Name \\
\hline$e=\lim _{n \rightarrow \infty}\left(1+\frac{1}{n}\right)^{n}\left(1+\frac{1}{2 n}\right)$ & $\mathrm{ACM}$ \\
\hline$e=\lim _{n \rightarrow \infty}\left(\frac{n+1}{n-1}\right)^{\frac{n}{2}}$ & MIM \\
\hline$e=\lim _{n \rightarrow \infty}(1+n)^{\frac{11 n}{6}}(n-1)^{\frac{5 n}{6}}\left(\frac{2 n+1}{2 n^{n+1}}\right)^{\frac{8}{3}}$ & ACMMIM \\
\hline$e=\lim _{n \rightarrow \infty}(1+n)\left(1+\frac{1}{n}\right)^{n}-(n-1)\left(1-\frac{1}{n}\right)^{-n}$ & B1 \\
\hline$e=\lim _{n \rightarrow \infty} \frac{2 n^{n}}{(2 n-1)(n-1)^{n-1}}$ & B2 \\
\hline$e=\lim _{n \rightarrow \infty} \frac{8}{7}(B 1)-\frac{1}{7}(M I M)$ & B5 \\
\hline$e=\lim _{n \rightarrow \infty} \frac{(1+n)^{1+n}}{2 n^{n}}+\frac{n^{n}}{(2 n-1)(n-1)^{n-1}}-\frac{n^{n}}{2(n-1)^{n-1}}$ & B3 \\
\hline$e=\lim _{n \rightarrow \infty} \frac{1}{6}(A C M)+\frac{5}{6}(B 2)$ & B4 \\
\hline$e=\lim _{n \rightarrow \infty} \frac{10}{7}(B 3)-\frac{3}{7}(B 4)$ & B6 \\
\hline$e=\lim _{n \rightarrow \infty} \frac{656}{75}(B 6)-\frac{581}{75}(B 5)$ & B7 \\
\hline
\end{tabular}

Table 2. Error (exact-formulae) by different closed form approximations for $n=100$.

\begin{tabular}{c|c||c|c}
\hline Formulae & Error & Formulae & Error \\
\hline $\boldsymbol{A C M}$ & $-5,607 \cdot 10^{-5}$ & $\mathbf{2 P - G L E E}$ & $-1,480 \cdot 10^{-10}$ \\
\hline MIM & $-9,062 \cdot 10^{-5}$ & $\mathbf{3 P - G L E E}$ & $-9,422 \cdot 10^{-16}$ \\
\hline ACMMIM & $1,507 \cdot 10^{-6}$ & $\|$ 4P-GLEE & $-5,923 \cdot 10^{-21}$ \\
\hline B6 & $3,902 \cdot 10^{-10}$ & $\|$ 5P-GLEE & $-3,702 \cdot 10^{-26}$ \\
\hline B7 & $-1,385 \cdot 10^{-10}$ & & \\
\hline
\end{tabular}




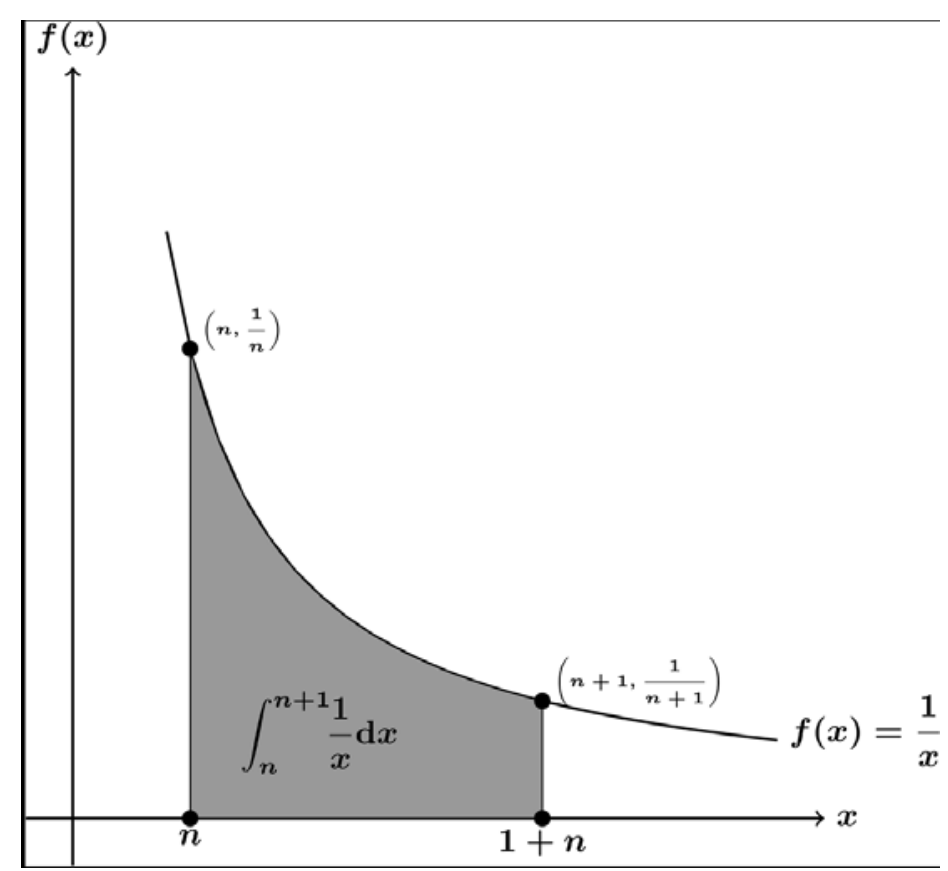

Figure 1. Graph of $f(x)=\frac{1}{x}$. The shaded area is equal to $\ln \left(1+\frac{1}{n}\right)$. 